\title{
$\mu$-PSEUDO ALMOST AUTOMORPHIC MILD SOLUTIONS FOR TWO TERMS ORDER FRACTIONAL DIFFERENTIAL EQUATIONS
}

\author{
Moumini Kéré And Gaston MANDATA N'GUÉRÉKATA
}

\begin{abstract}
In this paper we study the existence and uniqueness of $\mu$-pseudo almost automorphic mild solutions for two term fractional order differential equations in a Banach space with $\mu$-pseudo almost automorphic forcing terms. The fractional derivative is understood in the sense of Weyl. We use classical tools to obtain our results.
\end{abstract}

Mathematics subject classification (2010): 34M10, 30D35.

Keywords and phrases: $\mu$-pseudo almost automorphic functions, sectorial operator, fractional derivative.

\section{REFERENCES}

[1] A-N. Akdad, B. Es-Sebar, K. EzZinbi, Composition theorems of Stepanov $\mu$-pseudo almost automorphic functions and applications to nonautonomous neutral evolution equations, Differ. Equ. Dyn. Syst., Vol. 25 (2017), no. 3, 397-416.

[2] E. Alvarez-Pardo, C. LizAma, Weighted pseudo almost automorphic mild solutions for two term fractional order differential equations, Appl. Math. Comput., Vol. 271 (2015), 154-167.

[3] W. ARendt, C. BAtTy, M. Hieber, F. Neubrander, Vector-valued Laplace transforms and Cauchy problems, Monogr. Math., Vol. 96 (2001).

[4] J. Blot, P. Cieutat, K. Ezzinbi, Measure theory and pseudo automorphic functions: New developements and applications, Nonlinear Analysis 75 (2012), 2426-2447.

[5] J. Blot, P. Cieutat, G. M. N'Guérékata, D. Pennequin, Superposition operators between various almost periodic function spaces and applications, Commun. Math. Anal., Vol. 5 (2008), no. $2,42-70$.

[6] J. Blot, G. M. Mophou, G. M. N'Guérékata, D. Pennequin, Weighted pseudo almost automorphic functions and applications to abstract differential equations, Nonlinear Anal., Vol. 71 (2009), no. (3-4), 903-909.

[7] S. BochneR, Uniform convergence of monotone sequence of functions, Proc. Nat. Acad. Sci. U.S.A., Vol. 47 (1961), 582-585.

[8] S. Bochner, A new approach to almost periodicity, Proc. Nat. Acad. Sci. U.S.A., Vol. 48 (1962), 2039-2043.

[9] S. Bochner, Continuous mappings of almost automorphic and almost periodic functions, Proc. Nat. Acad. Sci. U.S.A., Vol. 52 (1964), 407-410.

[10] Y. CHANG, R. ZHANG, G. M. N'GUÉRÉKATA, Weighted pseudo almost automorphic mild solutions to semilinear fractional differential equations, Comput. Math. Appl., Vol. 64 (2012), 3160-3170.

[11] El H. A. DAdS, K. EzZinBi, M. MiraOUi, $(\mu, v)$-pseudo almost automorphic solutions for some non-autonomous differential equations, Internat. J. Math. Vol. 26 (2015), no. 11.

[12] M.A. Diop, K. EzZINBI, M.M. MBAYE, Existence and global attractiveness of a square-mean $\mu$ pseudo almost automorphic solutions for some stochastic evolution equation driven by Lévy noise, Math. Nachr., Vol. 290 (2017), no. 8-9, 1260-1280.

[13] V. Keyantuo, C. Lizama, M. Warma, Asymptotic behavior of fractional order semilinear evolution equations, Differential Integral Equations, Vol. 26 (2013), no. 7-8, 757-780. 
[14] J. Liang, G. M. N'GuéréKata, T-J. XiAo, J. Zhang, Some properties of pseudo almost automorphic functions and applications to abstract differential equations, Nonlinear Anal., Vol. 70 (2009), no. 7, 2731-2735.

[15] J. Liang, J. Zhang, T-J. Xiao, Composition of pseudo almost automorphic and asymptotically almost automorphic functions, J. Math. Anal. Appl., Vol. 340 (2008), no. 2, 1493-1499.

[16] K. S. Miller, B. Ross, An introduction to the fractional calculus and fractional differential equations, Wiley, New York, 1993.

[17] G. M. N'GUÉRÉKATA, Quelques remarques sur les fonctions asymptotiquement presque automorphes, Ann. Sci. Math. quebec, VII (1983) 185-191.

[18] G. M. N'GuÉRÉKATA, Almost automorphy and almost periodicity of motions in Banach spaces, Forum Math., Vol. 13 (2000), 581-588.

[19] G. M. N'GuÉRÉKATA, Topics in almost automorphy, Springer, New York 2004.

[20] G. M. N'GUÉRÉKATA, Spectral theory for bounded functions and applications to evolution equations, Nova Sciences Publishers, New York 2017.

[21] W. A. VeECH, Almost automorphic functions on groups, Amer. J. Math., Vol. 87 (1965), no. 3, 719 751.

[22] T.-J. Xiao, J. Liang, J. Zhang, Pseudo almost automorphic solutions to semilinear differential equations in Banach spaces, Semigroup Forum, Vol. 76 (2008), no. 3, 518-524.

[23] M. ZAKI, Almost automorphic solutions of certain abstract differential equations, Annali di Mat. Pura ed Appl., Vol. 101 (1974), no. 4, 91-114. 